\title{
Epigenetic Signatures as Biomarkers of Exposure
}

\author{
Christine Ladd-Acosta ${ }^{1}$
}

Published online: 14 April 2015

(C) Springer International Publishing AG 2015

\begin{abstract}
To advance our knowledge of the influence of environmental exposures on human health and disease, robust studies are needed. However, for many exposures, robust studies are not feasible due to limitations with current ascertainment methods and/or study designs. Epigenetics, the study of mitotically heritable, reversible information that regulates critical cell processes, has gained much attention because it offers a potential mechanism to explain how exposures can influence cell states. Therefore, most studies have focused on epigenetics as a mechanism for disease. However, emerging evidence also suggests that epigenetic marks may also serve as biomarkers of exposure. Here, we highlight findings showing that the epigenome is labile to the environment and that these exposure-associated changes show long-term stability, are specific, are detectable in accessible tissues, can predict exposure status, and can be practically implemented, thus supporting the potential for epigenetic patterns to serve as robust measures of environmental exposure.
\end{abstract}

Keywords Epigenetic $\cdot$ Environmental exposure · Biomarker $\cdot$ DNA methylation $\cdot$ Persistence $\cdot$ Signature

This article is part of the Topical Collection on Environmental Epigenetics

Christine Ladd-Acosta

claddac1@jhu.edu

1 Department of Epidemiology, Johns Hopkins Bloomberg School of Public Health, 615 N. Wolfe Street, E6518, Baltimore, MD 21205, USA

\section{Introduction}

Understanding the contribution of environmental exposures to human disease is a major focus of environmental health sciences and epidemiology and has important public health implications. Environmental exposures are typically ascertained using a broad range of collection instruments such as questionnaires, national registries, personal monitoring devices, geographic information systems, and biomarkers of exposure, i.e., chemical concentrations measured in biological fluids, which are obtained in a prospective or retrospective manner. Many of these collection methods have been shown to be highly robust and reliable for certain exposures, particularly when ascertained prospectively; however, for many studies, there are real practical barriers to obtaining reliable measures of exposure using existing methods. This is particularly problematic for exposures with short half-lives, studies interested in specific time frames where prospective ascertainment and biosample collection are not possible and retrospective exposure data is unreliable due to long recall times and/or recall biases, and exposure measurement that does not directly measure exposure levels present in individuals, i.e., effective biological dose, such as exposure estimates derived from geographic information systems. Even for exposures that can be reliably obtained using existing methods, exploring alternative methods of ascertainment is worthwhile because they may also be reliable and could provide a more cost-effective measure of exposure than the existing instruments.

Recent work highlights the potential for epigenetics, mitotically heritable, and reversible cellular information to fill this gap by serving as a robust molecular biomarker of exposure. Most studies seeking to investigate the role of epigenetics in environmental exposure and human disease have focused on epigenetics as a mechanism for disease, i.e., an environmental exposure leads to an epigenetic change that causes disease 
(Fig. 1a). However, recent evidence suggests that exposurerelated epigenetic changes may also serve as a proxy for exposure when investigating exposure-disease relationships in lieu of having actual prenatal exposure data (Fig. 1b). Thus, epigenetics may inform environmental health and epidemiology studies in two equally impactful ways (Fig. 1). The focus of this review is to summarize the evidence in humans that supports the potential for epigenetic signatures, i.e., patterns, to serve as biomarkers of environmental exposure (Fig. 1b).

\section{Epigenetic Modifications Are Environmentally Labile}

Epigenetics provides a mechanism for cells, with the same static genetic code, to develop into functionally distinct cell types and to change their cellular program in response to their environment. There are many different types of epigenetic marks including DNA methylation, histone tail modifications, small non-coding RNAs, and higher-order chromatin/nuclear structures. A central property of epigenetic data is that it is reversible, making it an attractive biological mechanism to link environmental exposures to cell alterations. In fact, changes in DNA methylation (DNAm), non-coding RNAs, and histone tail modifications have all been shown to be associated with human environmental exposures across a wide range of domains including toxicant, social/behavioral, diet, and pharmacologic. DNA methylation has been shown to undergo both losses and gains in global and/or repetitive elements with exposure to metals [1-3], benzene [4], persistent organic pollutants $[5,6]$, particulate matter $[7,8]$, mycotoxin [9], endocrine-disrupting chemicals [10], lifestyle factors [11, $12]$, and inorganic arsenic $[13,14]$. In addition, locus-specific differences in DNAm associated with exposure to nutrition [15-17], inorganic arsenic [18, 19], medications [20, 21], childhood abuse [22] and stress [23], socioeconomic status $[24,25]$, tobacco $[26-32,33 \cdot, 34,35 \cdot 36]$, polycyclic

\section{(A) Epigenetics as a MECHANISM}

$$
\text { Exposure } \longrightarrow \text { Epigenetics } \longrightarrow \text { Disease }
$$

\section{(B) Epigenetics as a BIOMARKER}

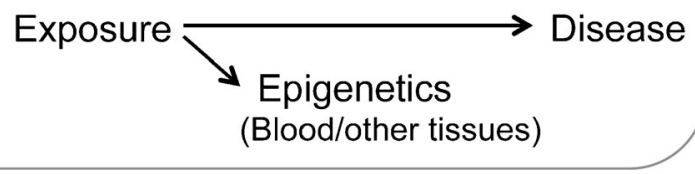

Fig. 1 Framework describing how epigenetic marks can inform environmental health and disease based research. a Epigenetics may provide a biological mechanism for environmental exposure associations with human disease. b Epigenetics may serve as a biomarker of environmental exposure aromatic hydrocarbons [37, 38], infections [39, 40], and endocrine-disrupting chemicals $[41,42]$ have also been observed. Changes in small non-coding RNAs have been found to be associated with inorganic arsenic [43] and ozone [44] exposures. Global changes in histone tail modifications have been shown to be associated with exposure to metals [45] and particulate matter [46, 47].

While exposure associations have been found for many different types of epigenetic marks, the evidence presented below, supporting the potential utility of epigenetics as a biomarker of exposure, mainly converges on DNAm for several reasons. First, DNAm is the most widely studied epigenetic mark; thus, given the recent emergence of this field, it has accumulated the most evidence to date compared to other types of epigenetic data. Second, DNAm has the potential to retain exposure signatures years later since it is the only epigenetic mark with a clear mechanism for post-mitotic inheritance. It is possible that some locus-specific DNAm marks in the genome are relatively stable, such as those involved in tissue specificity, while others are more labile. Recent evidence has shown that inter-individual variation in DNAm signatures is relatively stable in adulthood [48]. Although much work remains to determine which environmentally induced DNAm changes are rapid and transient and which are more stable over time, DNAm does offer an inherent biological mechanism for cells to remember environmental exposurerelated changes. Finally, at the current time, there are practical limitations to considering histone tail modifications or RNA as biomarkers of exposure. Histone tail modification measurements require very large numbers of cells and rapid DNAprotein cross-linking that is not practical for most large population-scale studies. RNA can degrade quickly, and the quality of RNA in many existing biosample repositories is likely to be poor; therefore, its utility as a biomarker is also questionable. DNA methylation, on the other hand, is stable and can be reliably measured from biospecimens that have been stored for many decades. Finally, as described in more detail below and in Table 1, existing technologies to measure DNAm are reproducible, cost-efficient, and amenable to highthroughput processing.

\section{Ideal Properties of a Biomarker of Exposure}

There is a growing body of literature showing that environmental exposures can influence the epigenome in humans. However, that alone does not merit its use as a biomarker. For an exposure biomarker to be useful it should (1) have a relatively long half-life that is suitable for a particular type of exposure and/or type of experiment, e.g., toxicants with acute effects may show DNAm signatures that are stable for hours or days, whereas chronic effects of exposure on DNAm may be present after many years; (2) show specificity; (3) be 
Table 1 Overview of locus-specific epigenetic measurement tools

\begin{tabular}{|c|c|c|c|c|c|c|}
\hline Scale & Epigenetic mark & Measurement tool $^{\mathrm{a}}$ & Material required & $\begin{array}{l}\text { Cost } \\
\text { (per sample) }\end{array}$ & Advantages ${ }^{\mathrm{b}}$ & Disadvantages $^{\mathrm{b}}$ \\
\hline \multirow[t]{3}{*}{ Genome-wide } & DNA methylation & WGBS-seq & DNA $(1 \mu \mathrm{g})$ & $1000 \mathrm{~s}$ of dollars & - Comprehensive coverage & - Expensive \\
\hline & Histone modifications & ChIP-seq & $\begin{array}{l}100 \text { s of thousands } \\
\text { of fresh cells }\end{array}$ & 1000 s of dollars & - Comprehensive coverage & $\begin{array}{l}\text { - Antibody specificity } \\
\text { - Type of biospecimen } \\
\text { - Expensive }\end{array}$ \\
\hline & RNA & RNA-seq & RNA $(1 \mu \mathrm{g})$ & 1000 s of dollars & - Comprehensive coverage & $\begin{array}{l}\text { - RNA instability } \\
\text { - Expensive }\end{array}$ \\
\hline \multirow[t]{3}{*}{ Genome-scale } & DNA methylation & Infinium $450 \mathrm{~K}^{\mathrm{c}}$ & DNA (500 ng) & 100 s of dollars & $\begin{array}{l}\text { - Cost } \\
\text { - Good coverage } \\
\text { - Stable biospecimen }\end{array}$ & - Genomic coverage \\
\hline & Histone modifications & ChIP-chip & $\begin{array}{r}\text { 10s of millions } \\
\text { of fresh cells }\end{array}$ & 100s of dollars & $\begin{array}{l}\text { - Cost } \\
\text { - Good coverage }\end{array}$ & $\begin{array}{l}\text { - Antibody specificity } \\
\text { - Type of biospecimen } \\
\text { - Genomic coverage }\end{array}$ \\
\hline & RNA & Expression arrays & RNA (350-500 ng) & 100 s of dollars & $\begin{array}{l}\cdot \text { Cost } \\
\cdot \text { - Good coverage }\end{array}$ & $\cdot$ RNA instability \\
\hline \multirow[t]{3}{*}{$\begin{array}{l}\text { Candidate } \\
\text { loci-scale }\end{array}$} & DNA methylation & $\begin{array}{l}\text { Bisulfite } \\
\text { pyrosequencing/ } \\
\text { EpiTyper }\end{array}$ & DNA (500 ng) & 10s of dollars & $\begin{array}{l}\text { - Inexpensive } \\
\text { - Stable biospecimen }\end{array}$ & - Genomic coverage \\
\hline & Histone modifications & ChIP-PCR & $\begin{array}{l}100 \text { s of thousands } \\
\text { of fresh cells }\end{array}$ & 100s of dollars & & $\begin{array}{l}\text { - Antibody specificity } \\
\text { - Type of biospecimen } \\
\text { - Cost } \\
\text { - Genomic coverage }\end{array}$ \\
\hline & RNA & TaqMan assays & RNA $(1-2 \mu \mathrm{g})$ & $100 \mathrm{~s}$ of dollars & & $\begin{array}{l}\text { - RNA instability } \\
\text { - Cost } \\
\text { - Genomic coverage }\end{array}$ \\
\hline
\end{tabular}

ChIP-seq chromatin immunoprecipitation sequencing, ChIP-chip chromatin immunoprecipitation sequencing, ChIP-PCR chromatin immunoprecipitation polymerase chain reaction, $R N A$-seq RNA sequencing, $W G B S$-seq whole-genome bisulfite sequencing

${ }^{a}$ Not an exhaustive list but is meant to represent the range of available assays with similar properties for biomarker purposes

${ }^{\mathrm{b}}$ From an exposure biomarker perspective

${ }^{\mathrm{c}}$ Illumina Infinium HumanMethylation450 BeadChip

present in an accessible tissue and reflect exposure dose; (4) be able to classify individuals based on their exposure status; and (5) be relatively inexpensive to measure in a large number of samples. While this area of research is still in its infancy and considerable work remains, in Table 2 and the sections below, we summarize current findings that support the potential for DNAm to serve as a robust exposure biomarker, i.e., describe how it meets each of these criteria.

\section{Exposure-Related DNA Methylation Changes Show Stability}

The existence of a stable epigenetic signature of exposure could overcome existing limitations for exposures with short half-lives. For example, the half-life of cotinine and phthalate monoesters is less than $24 \mathrm{~h}$, making past exposure difficult to assess, and cost-effective prospective studies are challenging since they are likely to require biospecimen collection at multiple time points. A biomarker of cumulative exposure would be beneficial for studies that seek to investigate the relationships between lifetime exposures and disease. For example, an epigenetic signature that reflects cumulative exposure to smoking may aid studies investigating the relationship between lifetime smoking exposure and lung cancer risk. Finally, epigenetic signatures of exposure may also serve as stable and practical biomarkers of rapid, acute changes related to environmental exposure, even if only present for hours or days after exposure. Several recent studies have shown that epigenetic changes associated with environmental exposures can be observed for months to years after exposure, offering a potential solution to this problem.

It is often difficult to study cumulative exposure because existing biomarkers for readily accessible tissues typically reflect short-term exposure levels. For example, lead in the blood has a half-life of 36 days; therefore, blood lead measurements reflect recent lead exposure. Bone lead reflects several years of exposure; therefore, cumulative lead exposure levels are currently obtained using bone scans, via K-X-ray fluorescence. Although these scans provide reliable estimates of several years worth of lead exposure, they can be costly and burdensome to research participants. DNA methylation levels at long-interspersed element 1 (LINE-1) elements in the blood 
Table 2 Summary of evidence supporting DNA methylation as a biomarker of exposure

\begin{tabular}{|c|c|}
\hline $\begin{array}{l}\text { Biomarker } \\
\text { property }\end{array}$ & Supporting citation(s) \\
\hline \multicolumn{2}{|l|}{ Stability } \\
\hline Months-years & $\begin{array}{l}\text { Vilahur et al. 2014; LaRocca et al. 2014; Koesteler } \\
\text { et al. 2013; Vidal et al. 2013; Youngblood et al. } \\
\text { 2013; Joubert et al. 2012; Kile et al. 2012; } \\
\text { Philibert et al. 2012; Smith et al. 2012; } \\
\text { Hoyo et al. 2011; Tobi et al. } 2009\end{array}$ \\
\hline Decades & $\begin{array}{l}\text { Breton et al. 2014; Zhang et al. 2014; Lee et al. } \\
\text { 2014; Mehta et al. 2013; Essex et al. 2013; } \\
\text { Borghol et al. 2012; Wright et al. } 2010\end{array}$ \\
\hline Persistence $^{\mathrm{a}}$ & $\begin{array}{l}\text { Lee et al. 2014; Novakovic et al. 2014; Youngblood } \\
\quad \text { et al. } 2013\end{array}$ \\
\hline \multicolumn{2}{|l|}{ Specificity } \\
\hline Exposure & $\begin{array}{l}\text { (Incomplete overlap of genomic regions across } \\
\text { studies with different exposures) }\end{array}$ \\
\hline Timing & $\begin{array}{l}\text { Wan et al. 2012; Tobi et al. } 2009 \text { (an incomplete } \\
\text { overlap of genomic regions across studies with } \\
\text { different exposure windows) }\end{array}$ \\
\hline \multicolumn{2}{|c|}{ Accurate classification } \\
\hline Dose-response & Zhang et al. 2014; Wright et al. 2010 \\
\hline Dichotomous & Shenker et al. 2013 \\
\hline Accessible tissue & $\begin{array}{l}\text { Goodrich et al. 2014; Essex et al. 2013; Vilahur } \\
\text { et al. 2014; LaRocca et al. 2014; Hoyo et al. } \\
\text { 2011; Joubert et al. 2012; Kile et al. 2012; } \\
\text { Koestler et al. 2013; Lee et al. 2014; Markunas } \\
\text { et al. 2014; Novakovic et al. 2014; Huen et al. } \\
\text { 2014; Kippler et al. 2013; Lee et al. 2014; Perera } \\
\text { et al. 2009; Smith et al. 2012; Tobi et al. 2009; } \\
\text { Vidal et al. 2013; Kim et al. 2013; Alegria-Torres } \\
\text { et al. 2013; Arita et al. 2012; Baccarelli et al. } \\
\text { 2009; Bailey et al. 2013; Bind et al. 2012; Bollati } \\
\text { et al. 2007; Borghol et al. 2012; Breitling et al. } \\
\text { 2011; Breton et al. 2014; Lambrou et al. 2012; } \\
\text { Lee et al. 2014; McGuinness et al. 2012; Mehta } \\
\text { et al. 2013; Philibert et al. 2012; Philibert et al. } \\
\text { 2010; Rusiecki et al. 2008; Shenker et al. 2013; } \\
\text { Sun et al. 2013; Tehranifar et al. 2013; } \\
\text { Tellez-Plaza et al. 2014; Terry et al. 2008; } \\
\text { Vineis et al. 2011; Wan et al. 2012; Wright et al. } \\
\text { 2010; Wu et al. 2013; Youngblood et al. 2013; } \\
\text { Zhang et al. 2014 }\end{array}$ \\
\hline $\begin{array}{l}\text { Existing } \\
\text { measurement } \\
\text { technologies: } \\
\text { inexpensive } \\
\text { and accurate }\end{array}$ & $\begin{array}{l}\text { Ehrich et al. 2005; Dupont et al. 2004; Eads et al. } \\
\quad 2000\end{array}$ \\
\hline
\end{tabular}

${ }^{\text {a }}$ Studies with longitudinal measures of DNA methylation

have been shown to be associated with patella bone lead levels, potentially offering a more attractive alternative measure of cumulative exposure for lead [49]. Similarly, studies on adults have shown that DNAm levels at specific smoking-associated loci reflect cigarette pack years and time since quitting [35•].
These findings provide support for the potential of DNAm to serve as a biomarker of cumulative exposure in adulthood.

In addition to showing associations in adulthood, DNA methylation changes related to prenatal exposures have been observed at birth and in early life. Joubert et al. first identified a 26-locus DNA methylation signature, in cord blood at birth, that is significantly associated with maternal plasma cotinine (a molecular biomarker of smoking) levels around gestational week 18 [50•]. Thus, showing smoking-related changes in DNAm can be observed about 22 weeks after the exposure window under investigation. DNAm levels at birth, from the cord blood or placenta, have also been associated with prenatal exposure to folate [17], inorganic arsenic [14, 19], phthalate and phenol during trimester 1 [41], medications [20, 21], caloric restriction [15], and xenoestrogens [10]. DNAm differences related to prenatal exposure have also been detected over much longer periods of time. For example, Breton et al. found significant associations between DNAm levels at 19 genomic loci obtained from the peripheral blood of children at age 12 and their prenatal exposure to smoking [36].

In addition to prenatal exposures, studies with DNA methylation measurements in adulthood have shown associations with early life exposures. For example, differences in DNAm at a few loci, detected using blood from adults over age 40, have been shown to be associated with exposure to abuse during childhood [22]. Preliminary studies have also shown that after adjustment for potential confounders, three classes of repetitive elements (Sat2, Alu, and LINE-1) among females and hundreds of specific genes in males [25] show DNAm differences in the adult peripheral blood that are related to their childhood socioeconomic status. Finally, exposure to stress in early childhood was found to be associated with site-specific changes in buccal cell DNAm in adolescents [23].

Two studies have directly assessed the persistence of prenatal smoking-associated DNAm changes, within an individual, over time. The first study took a candidate gene-based approach and found that DNAm changes in the AHRR gene related to prenatal smoking exposure are present at birth and are consistent with DNAm patterns in the same individuals at 18 months of age [28]. A second, larger, independent study examined DNAm patterns at five loci among individuals with a biospecimen collected at birth, age 7, and age 17 . They found that DNAm patterns at all five sites were significantly different and consistent across samples obtained at birth and age 7 between the prenatally exposed and unexposed individuals [51••]. Furthermore, four out of five sites showed similar patterns of DNAm at age 17 [51 • $]$. These findings remained significant even after adjusting for exposure to secondhand smoke and firsthand smoking during childhood and adolescence $[51 \bullet \bullet]$.

Persistent changes in DNA methylation have also been observed for other exposures as well as for both child and adulthood exposure windows. For example, during acute 
exposure (with a high viral load) to human immunodeficiency virus (HIV), virus-specific CD8 positive T cells undergo loss of DNA methylation at the PD-1 locus [40]. This DNAm change has been shown to persist in chronic stages of infection, even after the viral load is reduced to undetectable levels using highly active antiretroviral therapy (HAART) [40].

\section{Specificity of Epigenetic Signatures of Exposure}

For an epigenetic signature to be a useful biomarker of exposure, it should be specific for a particular exposure under investigation. Additionally, it would be useful to have epigenetic biomarkers that are specific to different windows of exposure and/or provide information about changes in exposure status at different points in time.

\section{Exposure-Specific Signatures}

Given the recent emergence of this field, little work has been done to date to directly address the specificity of exposureassociated epigenetic signatures in humans. However, the lack of overlap among the specific loci and genomic regions identified by studies of different exposures provides some plausibility for the existence of exposure-specific genomic location differences. For example, none of the top ten $\mathrm{CpG}$ sites identified as associated with prenatal exposure to cadmium [52] were identified in studies of prenatal $[27,50 \bullet 51 \bullet \cdot]$ or adult $[31,32]$ exposure to smoking. Importantly, since these studies measured DNAm using the Illumina Infinium $450 \mathrm{~K}$ BeadChip, they were capable of identifying the same sites.

\section{Exposure Window-Specific Signatures}

Site-specific differences in DNA methylation at birth related to prenatal exposure to famine were shown to differ, depending on the gestational timing of the exposure [15]. Initial work in adults suggests that DNA methylation signatures of exposure to smoking, i.e., sets of loci, may differ based on whether the individual is a current, previous, or never smoker. A genome-scale screen of DNAm, using adult peripheral blood samples, revealed 15 loci with significant differential methylation related to levels of cumulative exposure to tobacco smoke (in pack years), current exposure to active smoking, and former smokers, compared to individuals that never smoked [33•]. Among the group of former smokers, DNA methylation levels at three sites were significantly associated with time since quitting, with increasing amounts of DNAm related to longer times since quitting. Although these three sites also came up as associated with current smoking exposure, the direction of change in DNAm differed [33•]; thus, these three loci show window-specific differences in DNAm alterations.
Additional evidence supporting the potential for DNAm signatures to be specific to a particular exposure period comes from the lack of complete overlap between sites identified in prenatal versus adult exposure windows for smoking, even though the sample sizes for each exposure time frame are comparable. For example, two of the strongest genomic regions, in the F2RL3 and LRP5 genes, identified and replicated in adult studies as associated with smoking exposure [26, 31, $32,33 \cdot, 35 \cdot$ have not been identified in any of the newborn or childhood studies of prenatal smoking exposure $[27,36,50$, $51 \bullet \bullet$. In fact, Markunas et al. recently compared genomic regions showing differential DNAm associations with smoking across multiple adult studies to genomic regions showing DNAm alterations associated with prenatal exposure to smoking. For the four genes (AHRR, GNG12, GFI1, and CNTNAP2), smoking-related DNAm alterations were present in both the adult and prenatal exposure windows [27]. However, $11 / 17$ and 6/10 genomic regions were restricted to the prenatal and adult exposure windows, respectively [27]. These findings highlight that some changes in DNAm may be common across different exposure windows; however, many appear to be specific for a particular exposure window.

\section{Accurate Prediction of Exposure Using Epigenetic Measurements}

A critical component of any biomarker is the ability to accurately classify samples into exposure categories and/or provide a quantitative measure of exposure for each sample. DNA methylation levels are quantitative in nature, with values ranging from 0 to $100 \%$; thus, they are inherently well suited to report quantitative measures of exposure. Empirical evidence for DNAm as a quantitative measure of exposure stems from recent work detailing dose-response relationships in adults with smoking exposure and DNAm levels at a locus in the F2RL3 gene [35•]. For current smokers, the authors observed a strong inverse relationship between DNAm levels and the average number of cigarettes smoked per day [35•]. Among former smokers, they report a dose-response relationship between methylation at F2RL3 and time since quitting smoking, up to about 20-25 years; individuals with longer times since smoking cessation had higher levels of DNAm [35•]. Studies have also shown dose-response associations between cumulative measures of lifetime exposure to smoking [35•] and lead and DNAm [49].

In addition to dose-response relationships, DNAm levels at smoking exposure-related $\mathrm{CpG}$ sites have been assessed for their ability to accurately classify samples into dichotomous exposure categories. A classifier built using DNA methylation values at four smoking-associated loci, measured 14 years after smoking cessation, on average, was able to predict former and never smokers with $71 \%$ sensitivity and an area 
under the curve (AUC) value, a measure of classification accuracy, of 0.83 [53••]. In comparison, a predictive model built to classify former and never smokers using cotinine measurements, a widely used measure of smoking exposure, showed poorer performance with an AUC value of 0.47 [53••].

\section{Practical Considerations}

\section{Detectable in Accessible Tissues}

Examination of affected tissues is important for studies that seek to provide a mechanistic link between an exposure and disease; however, they are not needed for biomarker purposes. In fact, for a biomarker to be useful, it should be detectable and should reflect exposure levels in a readily accessible tissue such as blood, saliva, or buccal mucosa. Since blood circulates throughout the body and comes into contact with all organ systems, it is plausible that exposures with various routes of entry may come into contact with blood. Studies have shown that inter-individual differences in DNAm are highly consistent across tissues $[54,55]$. Although some of these interindividual differences are likely to be driven by underlying genetic variation, there is evidence that some genomic regions with changes in DNAm related to differences in periconceptional nutrition status are also maintained across tissues [56]. Finally, the most compelling evidence that DNAm changes, with values that reflect exposure levels, which can be detected in accessible tissues, is empirical: all of the studies described in the preceding sections of this review were carried out using surrogate tissues including buccal mucosal cells $[3,23]$, placenta $[10,41]$, cord blood $[5,14,15$, $17,19-21,27,28,38,50 \bullet, 51 \bullet \bullet, 52]$, saliva [42], and peripheral blood cells $[2,4,6-9,11-13,16,18,22,24-26,29-32$, $33 \bullet, 35 \bullet, 36,37,40,45,49,51 \bullet \bullet$.

\section{Availability of Cost-Effective Epigenetic Measurement Tools}

There are a large number of reproducible and accurate genome-wide, site-specific, and global assays available to measure DNA methylation, each with their own advantages and disadvantages with respect to cost and sample throughput [57-59]. It is likely that more expensive genome-wide approaches, costing hundreds to thousands of dollars per sample, will be needed to initially identify and define exposurespecific epigenomic signatures, i.e., sets of loci (Table 1). However, once these signatures have been established, there are a number of highly accurate, reproducible, and inexpensive technologies such as bisulfite pyrosequencing [60], MethyLight [61], and EpiTyper [58] available to measure a subset of genomic sites that comprise a particular exposure signature (Table 1). These cost an order of magnitude less that genome-wide technologies, costing tens of dollars per sample, and are well within (or below) the cost range of other commonly used exposure biomarkers such as cotinine and metal serum measurements (Table 1).

\section{Challenges and Future Directions}

We have highlighted several lines of evidence showing the promise of epigenetic signatures as biomarkers of exposure; however, there are also many challenges. Because DNA methylation patterns at some loci in the genome are cell type specific and tissues are heterogeneous, it is possible that differences in DNA methylation shown to be associated with environmental exposures may simply reflect shifts in the proportions of specific cell types in a given tissue [62]. For example, Kile et al. found that prenatal exposure to arsenic prior to 16 weeks of gestation was associated with an increase and decrease in cord blood CD8-positive and CD4-positive T cells, respectively [63]. Due to this concern, analytic methods [64-66] have recently emerged and are being widely applied in epigenomic studies to account for potential shifts in underlying cell proportions. While it is important to understand the underlying cause of observed differences in DNAm, from a biomarker perspective, removal of potential confounding by cell type may be less of an issue than it is for mechanistic studies. Even if the observed DNAm differences associated with exposures merely reflect shifts in underlying cell proportions, DNAm could still serve as a biomarker of exposure, i.e., it would be an accurate, quantitative measure of shifts in cell proportions that are related to exposure.

It is also possible that epigenetic signatures of exposure may be influenced by genetic variation and/or geneenvironment interactions [67]. Future studies to define epigenomic changes related to exposures should also consider potential genetic modifiers. This is likely to require unified population-scale datasets with genetic, environmental, and epigenetic data from the same individuals and development of new integrative statistical approaches.

Most of the evidence provided above in support of the potential for epigenetics to serve as a biomarker of exposure has focused on tobacco smoke. While it is likely that these findings would extend to other exposures, at least in some cases, studies designed to rigorously evaluate the extension of these properties to other environmental exposures are needed. Similarly, additional studies to investigate exposure specificity, persistence, and the predictive potential of epigenomic signatures exposures are needed. These studies will likely involve close collaboration among researchers across several fields including, epidemiology, basic science, environmental health sciences, biostatistics, and epigenomics. In fact, collaborative efforts are already underway to begin to relate "omics" data to early life exposures through The Human Early-Life 
Exposome (HELIX) project [68]. Finally, it is important to consider the potential ethical and legal impact of a biomarker of exposure, including epigenetic signatures, to determine whether there are likely to be any unintended consequences as a result of their use [69].

\section{Conclusions}

In this review, we present evidence showing the potential for epigenetic signatures to serve as biomarkers of environmental exposures. These findings highlight that DNA methylation fulfills several ideal biomarker criteria including long-term robust changes associated with environmental exposures, exposure specificity, dose-response relationships, the ability to accurately predict exposure status that it is detectable in accessible tissues, and existing technologies to measure DNAm that are accurate, reliable, and relatively inexpensive. Should future studies definitively show that epigenetic signatures can be used in lieu of actual exposure data, they could overcome several limitations with existing exposure ascertainment methods. Thus, enabling new studies seeking to investigate the relationships between environmental exposures and human health may otherwise not be possible.

Acknowledgments The author would like to thank Dr. Daniele Fallin for her many thoughtful discussions on the subject of epigenetic epidemiology and epigenetic biomarkers. C. Ladd-Acosta is supported by the following grants: Autism Speaks award \#7659 (Fallin), R01ES017646 (Fallin/Feinberg), P50 HG003233 (Feinberg), NIH U01AI090727 (Wang), and R01AG042187 (Feinberg)

\section{Compliance With Ethics Guidelines}

Conflict of Interest Christine Ladd-Acosta declares that she has no conflict of interest.

Human and Animal Rights and Informed Consent This article does not contain any studies with human or animal subjects performed by any of the authors.

\section{References}

Papers of particular interest, published recently, have been highlighted as:

- Of importance

•. Of major importance

1. Li C et al. Epigenetic marker (LINE-1 promoter) methylation level was associated with occupational lead exposure. Clin Toxicol (Phila). 2013;51(4):225-9.

2. Tellez-Plaza M et al. Association of global DNA methylation and global DNA hydroxymethylation with metals and other exposures in human blood DNA samples. Environ Health Perspect. 2014;122(9):946-54.
3. Goodrich JM et al. Mercury biomarkers and DNA methylation among Michigan dental professionals. Environ Mol Mutagen. 2013;54(3):195-203.

4. Bollati V et al. Changes in DNA methylation patterns in subjects exposed to low-dose benzene. Cancer Res. 2007;67(3):876-80.

5. Huen $\mathrm{K}$ et al. Effects of age, sex, and persistent organic pollutants on DNA methylation in children. Environ Mol Mutagen. 2014;55(3):209-22.

6. Rusiecki JA et al. Global DNA hypomethylation is associated with high serum-persistent organic pollutants in Greenlandic Inuit. Environ Health Perspect. 2008;116(11):1547-52.

7. Bind MA et al. Air pollution and markers of coagulation, inflammation, and endothelial function: associations and epigeneenvironment interactions in an elderly cohort. Epidemiology. 2012;23(2):332-40

8. Baccarelli A et al. Rapid DNA methylation changes after exposure to traffic particles. Am J Respir Crit Care Med. 2009;179(7):572-8.

9. Wu HC et al. Global DNA methylation in a population with aflatoxin B1 exposure. Epigenetics. 2013;8(9):962-9.

10. Vilahur $\mathrm{N}$ et al. Prenatal exposure to mixtures of xenoestrogens and repetitive element DNA methylation changes in human placenta. Environ Int. 2014;71:81-7.

11. McGuinness D et al. Socio-economic status is associated with epigenetic differences in the pSoBid cohort. Int $\mathrm{J}$ Epidemiol. 2012;41(1):151-60

12. Terry MB et al. Genomic DNA methylation among women in a multiethnic New York City birth cohort. Cancer Epidemiol Biomarkers Prev. 2008;17(9):2306-10.

13. Lambrou A et al. Arsenic exposure and DNA methylation among elderly men. Epidemiology. 2012;23(5):668-76.

14. Kile ML et al. Prenatal arsenic exposure and DNA methylation in maternal and umbilical cord blood leukocytes. Environ Health Perspect. 2012;120(7):1061-6.

15. Tobi EW et al. DNA methylation differences after exposure to prenatal famine are common and timing- and sex-specific. Hum Mol Genet. 2009;18(21):4046-53.

16. Vineis $\mathrm{P}$ et al. DNA methylation changes associated with cancer risk factors and blood levels of vitamin metabolites in a prospective study. Epigenetics. 2011;6(2):195-201.

17. Hoyo $\mathrm{C}$ et al. Methylation variation at IGF2 differentially methylated regions and maternal folic acid use before and during pregnancy. Epigenetics. 2011;6(7):928-36.

18. Bailey KA et al. Arsenic and the epigenome: interindividual differences in arsenic metabolism related to distinct patterns of DNA methylation. J Biochem Mol Toxicol. 2013;27(2):106-15.

19. Koestler DC et al. Differential DNA methylation in umbilical cord blood of infants exposed to low levels of arsenic in utero. Environ Health Perspect. 2013;121(8):971-7.

20. Vidal $\mathrm{AC}$ et al. Associations between antibiotic exposure during pregnancy, birth weight and aberrant methylation at imprinted genes among offspring. Int J Obes (Lond). 2013;37(7):907-13.

21. Smith AK et al. Prenatal antiepileptic exposure associates with neonatal DNA methylation differences. Epigenetics. 2012;7(5): 458-63.

22. Mehta D et al. Childhood maltreatment is associated with distinct genomic and epigenetic profiles in posttraumatic stress disorder. Proc Natl Acad Sci U S A. 2013;110(20):8302-7.

23. Essex MJ et al. Epigenetic vestiges of early developmental adversity: childhood stress exposure and DNA methylation in adolescence. Child Dev. 2013;84(1):58-75.

24. Tehranifar P et al. Early life socioeconomic factors and genomic DNA methylation in mid-life. Epigenetics. 2013;8(1):23-7.

25. Borghol $\mathrm{N}$ et al. Associations with early-life socio-economic position in adult DNA methylation. Int J Epidemiol. 2012;41(1):62-74. 
26. Breitling LP et al. Tobacco-smoking-related differential DNA methylation: $27 \mathrm{~K}$ discovery and replication. Am J Hum Genet. 2011;88(4):450-7.

27. Markunas CA et al. Identification of DNA methylation changes in newborns related to maternal smoking during pregnancy. Environ Health Perspect. 2014;122(10):1147-53.

28. Novakovic B et al. Postnatal stability, tissue, and time specific effects of AHRR methylation change in response to maternal smoking in pregnancy. Epigenetics. 2014;9(3):377-86.

29. Philibert RA, Beach SR, Brody GH. Demethylation of the aryl hydrocarbon receptor repressor as a biomarker for nascent smokers. Epigenetics. 2012;7(11):1331-8.

30. Philibert RA et al. The effect of smoking on MAOA promoter methylation in DNA prepared from lymphoblasts and whole blood. Am J Med Genet B Neuropsychiatr Genet. 2010;153B(2):619-28.

31. Shenker NS et al. Epigenome-wide association study in the European Prospective Investigation into Cancer and Nutrition (EPIC-Turin) identifies novel genetic loci associated with smoking. Hum Mol Genet. 2013;22(5):843-51.

32. Sun YV et al. Epigenomic association analysis identifies smokingrelated DNA methylation sites in African Americans. Hum Genet. 2013;132(9):1027-37.

33. Wan ES et al. Cigarette smoking behaviors and time since quitting are associated with differential DNA methylation across the human genome. Hum Mol Genet. 2012;21(13):3073-82. This study observed three genomic loci showing exposure window-specific differences in DNA methylation related to smoking exposure.

34. $\mathrm{Xu} \mathrm{Q}$ et al. Determination of methylated $\mathrm{CpG}$ sites in the promoter region of catechol- $O$-methyltransferase (COMT) and their involvement in the etiology of tobacco smoking. Front Psychiatry. 2010;1:16.

35. Zhang $\mathrm{Y}$ et al. F2RL3 methylation as a biomarker of current and lifetime smoking exposures. Environ Health Perspect. 2014;122(2): 131-7. This study shows dose-response relationships between DNA methylation levels and number of cigarettes smoked per data as well as cumulative smoke exposure (in pack years).

36. Breton CV et al. Prenatal tobacco smoke exposure is associated with childhood DNA CpG methylation. PLoS One. 2014;9(6):e99716.

37. Alegria-Torres JA et al. Epigenetic markers of exposure to polycyclic aromatic hydrocarbons in Mexican brickmakers: a pilot study. Chemosphere. 2013;91(4):475-80.

38. Perera $\mathrm{F}$ et al. Relation of DNA methylation of $5^{\prime}-\mathrm{CpG}$ island of ACSL3 to transplacental exposure to airborne polycyclic aromatic hydrocarbons and childhood asthma. PLoS One. 2009;4(2):e4488.

39. Uddin $\mathrm{M}$ et al. Epigenetic and immune function profiles associated with posttraumatic stress disorder. Proc Natl Acad Sci U S A. 2010;107(20):9470-5.

40. Youngblood B et al. Cutting edge: prolonged exposure to HIV reinforces a poised epigenetic program for PD-1 expression in virus-specific CD8 T cells. J Immunol. 2013;191(2):540-4.

41. LaRocca $J$ et al. The impact of first trimester phthalate and phenol exposure on IGF2/H19 genomic imprinting and birth outcomes. Environ Res. 2014;133:396-406.

42. Kim JH et al. Bisphenol A-associated epigenomic changes in prepubescent girls: a cross-sectional study in Gharbiah. Egypt Environ Health. 2013;12:33.

43. Rager JE et al. Prenatal arsenic exposure and the epigenome: altered microRNAs associated with innate and adaptive immune signaling in newborn cord blood. Environ Mol Mutagen. 2014;55(3):196-208.

44. Fry RC et al. Air toxics and epigenetic effects: ozone altered microRNAs in the sputum of human subjects. Am J Physiol Lung Cell Mol Physiol. 2014;306(12):L1129-37.

45. Arita A et al. Global levels of histone modifications in peripheral blood mononuclear cells of subjects with exposure to nickel. Environ Health Perspect. 2012;120(2):198-203.

46. Cantone $\mathrm{L}$ et al. Extracellular histones mediate the effects of metal-rich air particles on blood coagulation. Environ Res. 2014;132:76-82.
47. Cantone $\mathrm{L}$ et al. Inhalable metal-rich air particles and histone $\mathrm{H} 3 \mathrm{~K} 4$ dimethylation and $\mathrm{H} 3 \mathrm{~K} 9$ acetylation in a cross-sectional study of steel workers. Environ Health Perspect. 2011;119(7):964-9.

48. Feinberg AP et al. Personalized epigenomic signatures that are stable over time and covary with body mass index. Sci Transl Med. 2010;2(49):49ra67.

49. Wright RO et al. Biomarkers of lead exposure and DNA methylation within retrotransposons. Environ Health Perspect. 2010;118(6):790-5.

50. Joubert BR et al. 450K epigenome-wide scan identifies differential DNA methylation in newborns related to maternal smoking during pregnancy. Environ Health Perspect. 2012;120(10):1425-31. This was the first genome-scale study showing that their findings have now been replicated in numerous independent samples.

$51 . \bullet$ Lee KW et al. Prenatal exposure to maternal cigarette smoking and DNA methylation: epigenome-wide association in a discovery sample of adolescents and replication in an independent cohort at birth through 17 years of age. Environ Health Perspect. 2014;123(2): 193-9. This study shows persistence of epigenetic patterns, in the same individuals, related to prenatal exposure to smoking up to age 17. These findings remained significant after adjusting for early life and adolescent smoking exposure. Several of these sites have also been replicated in birth cohorts.

52. Kippler $M$ et al. Sex-specific effects of early life cadmium exposure on DNA methylation and implications for birth weight. Epigenetics. 2013;8(5):494-503.

53.• Shenker NS et al. DNA methylation as a long-term biomarker of exposure to tobacco smoke. Epidemiology. 2013;24(5):712-6.

This study shows that previous exposure to smoking can be predicted using current DNA methylation measurements from blood samples.

54. Ma B et al. Predicting DNA methylation level across human tissues. Nucleic Acids Res. 2014;42(6):3515-28.

55. Davies $\mathrm{MN}$ et al. Functional annotation of the human brain methylome identifies tissue-specific epigenetic variation across brain and blood. Genome Biol. 2012;13(6):R43.

56. Waterland RA et al. Season of conception in rural gambia affects DNA methylation at putative human metastable epialleles. PLoS Genet. 2010;6(12):e1001252.

57. Irizarry RA et al. Comprehensive high-throughput arrays for relative methylation (CHARM). Genome Res. 2008;18(5):780-90.

58. Ehrich $\mathrm{M}$ et al. Quantitative high-throughput analysis of DNA methylation patterns by base-specific cleavage and mass spectrometry. Proc Natl Acad Sci U S A. 2005;102(44):15785-90.

59. Callinan PA, Feinberg AP. The emerging science of epigenomics. Hum Mol Genet. 2006;15(1):R95-101.

60. Dupont JM et al. De novo quantitative bisulfite sequencing using the pyrosequencing technology. Anal Biochem. 2004;333(1):119-27.

61. Eads CA et al. MethyLight: a high-throughput assay to measure DNA methylation. Nucleic Acids Res. 2000;28(8):E32.

62. Houseman EA et al. DNA methylation arrays as surrogate measures of cell mixture distribution. BMC Bioinforma. 2012;13:86.

63. Kile ML et al. Effect of prenatal arsenic exposure on DNA methylation and leukocyte subpopulations in cord blood. Epigenetics. 2014;9(5):774-82.

64. Accomando WP et al. Quantitative reconstruction of leukocyte subsets using DNA methylation. Genome Biol. 2014;15(3):R50.

65. Houseman EA, Molitor J, Marsit CJ. Reference-free cell mixture adjustments in analysis of DNA methylation data. Bioinformatics. 2014;30(10):1431-9.

66. Koestler DC et al. Blood-based profiles of DNA methylation predict the underlying distribution of cell types: a validation analysis. Epigenetics. 2013;8(8):816-26.

67. Teh AL et al. The effect of genotype and in utero environment on interindividual variation in neonate DNA methylomes. Genome Res. 2014;24(7):1064-74. 
68. Vrijheid M et al. The Human Early-Life Exposome (HELIX): project rationale and design. Environ Health Perspect. 2014;122(6): 535-44.
69. Miranda RC et al. A report on the fetal alcohol spectrum disorders study group meeting of 2012, theme title, "biomarkers for FASD". Alcohol. 2013;47(8):583-7. 